\title{
Tracking radiology's risks
}

$\mathrm{C}$ alling friends, checking email and playing games are par for the course for iPhone users.

But few likely expect their iPhone to calculate cancer risks from medical exams such as computed tomography (CT) scans.

That's exactly what a fourth-year radiology resident at the University of Toronto in Toronto, Ontario, hopes iPhone and iTouch users will do, using a $\$ 2.99$ application launched in May that uses available research to estimate patients' cumulative radiation doses as a result of medical exams and then calculates the associated cancer risk.

"The point of the application is to simply raise awareness among both patients and physicians about radiation risk associated with various radiology and radiology-related exams," says Dr. Mark Otto Baerlocher, who designed the application with his brother Adrian Baerlocher, a computer programmer.

The Radiation Passport program allows users to enter exam histories, medical instructions and appointments. It is similar in principle to a website operated by a group of radiology residents in the United States (www .xrayrisk.com).

Calculating the cumulative dose is a difficult task, given the many and sometimes conflicting studies, varying radiation doses and the different effects radiation can have depending on age and gender. But Baerlocher says that must change, given the rapid growth in use of CT exams, which use higher radiation doses. CT exam use grew nearly $28 \%$ between 2003/2004 and 2006/2007, and the number of CT scanners in Canada more than doubled between 1990 and 2007, according to the Canadian Institute of Health Information.

Various studies and reports have claimed, with some controversy, that as many as one-third of CT scans in the United States are unnecessary and that between $1 \%$ and $2 \%$ of cancer cases may be caused by CT radiation exposure.

A campaign called Image Gently,

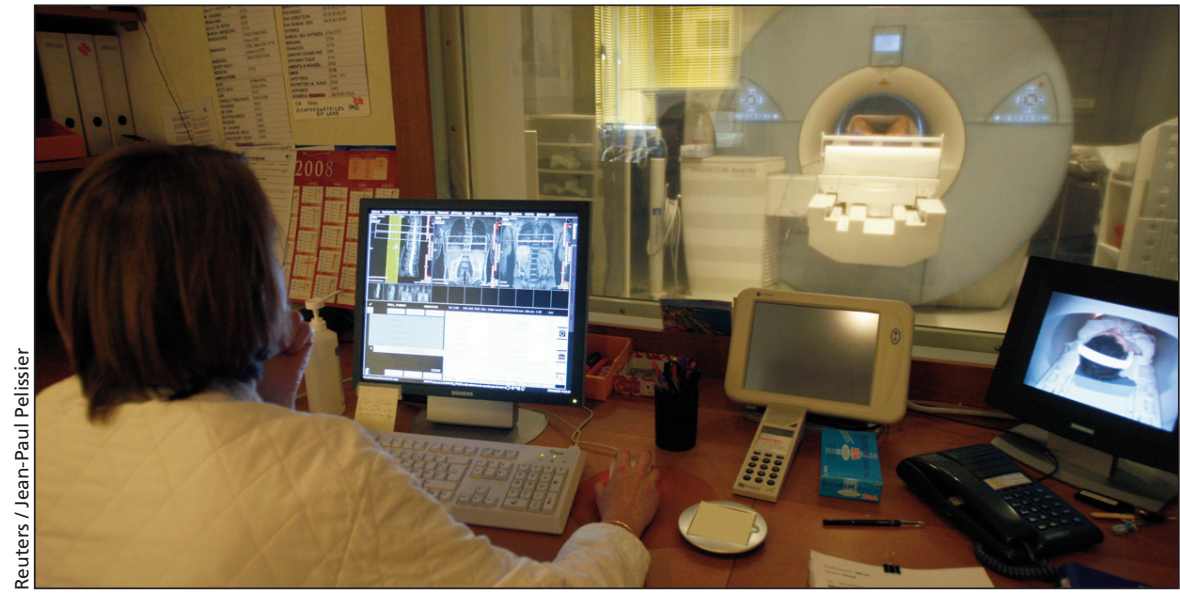

A radiologist studies an image from a magnetic resonance imaging scanner.

backed by several prominent medical bodies, including the American College of Radiology and the Canadian Association of Radiologists, also emerged, advocating the need to reduce radiation doses on children, among other issues.

Although supportive of efforts to increase awareness, some are concerned that tools such as Radiation Passport may not help patients understand the balance between the risks and benefits.

"Is it fair for patients to have that information and be responsible for processing it?" asks Dr. Donald Frush, chair of the American College of Radiology Pediatric Imaging Commission and a member of the Image Gently campaign. Frush says concerns about the Radiation Passport program were raised at a recent meeting of the International Atomic Energy Agency. "A patient may refuse a scan because they downloaded a program on their iPhone that tells them the risk is high, and they say 'forget it' when they've made the decision based only on the risks and not on the benefits."

Likewise, the Canadian Association of Radiologists is promoting an approach called "the best test first," which relies on standards and guidelines to judge when a test is necessary.

The association is involved in projects at 2 Manitoba hospitals to create a program that recommends necessary tests based on the patient's condition. It would differ from Radiation Passport in that it would be a centralized system that offers recommendations along with the advice of a medical worker.

Dr. Ted Lyons, president of the Canadian Association of Radiologists, says a program such as Radiation Passport risks generalizing cancer risks while confusing patients and doctors alike. "Bottom line is, 1 abdominal CT scan is equal to 500 chest X-rays [in terms of radiation dose]," he says. "If you need the test, the CT scan should be done. But in patients who need continual monitoring, should you do repeat $\mathrm{CT}$ scans on a regular basis, or because of the high radiation dose, are there better tests to do in the interim?"

Although there may be some disagreement about the best way to approach the issue, Baerlocher says the end result is what's most important greater discussion and understanding of the effects of radiation.

"If you're an emergency physician and all of a sudden some of your patients start to come to you asking about the associated risks of CT scans and they don't know them, they'll likely start looking them up pretty quickly," Baerlocher says. - Christopher Mason, Ottawa, Ont.

DOI:10.1503/cmaj.091049 